\title{
Gastrointestinal Stromal Tumor Associated with Neurofibromatosis Type 1 Simulating a Neurofibrosarcoma in a Black African Adult Patient
}

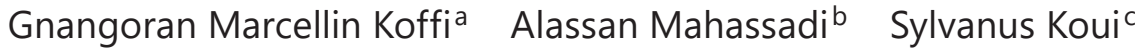 \\ Kountele Gona Soro ${ }^{a}$ \\ aDepartment of Visceral surgery, Faculty of Medicine, Felix Houphouet Boigny University, \\ Yopougon Teaching Hospital, Abidjan, Ivory Coast; 'bepartment of Gastroenterology, \\ Felix Houphouet Boigny University, Yopougon Teaching Hospital, Abidjan, Ivory Coast; \\ 'Department of Pathology, Felix Houphouet Boigny University, Treichville Teaching Hospital, \\ Abidjan, Ivory Coast
}

\section{Keywords}

Intestinal bleeding · Gastrointestinal stromal tumor - Neurofibromatosis - Africa

\begin{abstract}
Gastrointestinal stromal tumor (GIST) is the most prevalent mesenchymal tumor encountered in patients with neurofibromatosis type I (NF1). This association is rarely reported in black African adult patients. Clinical signs such as abdominal pain, intestinal bleeding, and palpable abdominal nodules may contribute to the diagnosis of GIST. However, these clinical signs may also indicate the presence of other solid intestinal tumors such as a neurofibrosarcoma which is an intestinal complication of NF1. The immunohistochemistry staining of the resected tissue is mandatory that allows establishing a correct diagnostic of GIST. The immunohistochemistry is also a challenging method in African countries with limited resources. Herein, we report a case of multiple intestinal involvement of GIST associated with NF1 revealed by a recurrent intestinal bleeding in a black African adult patient. The GIST diagnosis was suspected on a computed tomography scan images and laparotomy findings, which were confirmed by histopathological and immunochemistry examinations of the resected nodules. The immunohistochemistry staining of the tissue was positive for CD34 and CD117 indicating the presence of GIST in black African patient with NF1. The surgical treatment was consisted of a double intestinal resection with anastomosis that removed all palpable nodules located on the intestinal antimesenteric wall. No additional chemotherapy was administered to the patient who is so far under follow-up. The patient has not presented any episode of intestinal bleeding since
\end{abstract}


the surgical treatment. We emphasize in this case report the importance of a recurrent intestinal bleeding in patient with NF1 that may indicate the presence of GIST and, the need of performing a large intestinal resection, as the most valuable treatment in limited resource countries.

(C) 2021 The Author(s)

Published by S. Karger AG, Basel

\section{Introduction}

Gastrointestinal stromal tumor (GIST) is the most common mesenchymal tumor associated with neurofibromatosis type 1 (NF1) occurring in $20 \%$ of cases. NF1 is an autosomal dominant genetic disease, also known as Von Recklinghausen disease. Approximately 250 patients have been described worldwide [1, 2]. The GIST may be asymptomatic or in some cases, characterized by bleeding, intussusception, abdominal pain, or palpable mass [3-5]. GIST could share clinical manifestations with various neurogenic or mesenchymal intestinal tumors such as a neurofribrosarcoma occurring in patient with NF1; however, the histopathological patterns of these tumors are quite different from that of GIST [3-7]. Diagnostic criteria of NF1 are well established and based on the presence of 2 among the following 7 criteria: $>6$ spots "café au-lait" of at least $15 \mathrm{~mm}$ in diameter, freckling in the axillary or inguinal region, at least 2 neurofibroma different types or at least one plexiform neurofibroma or optic nerve glioma, $\geq 2$ iris Lisch nodules, bone lesions such as pseudarthrosis, sphenoid wing dysplasia and long bone cortex thinning, and relative or parents of first-degree cases [8]. However, NF1 is reported in black Africans and its association with GIST is rarely described [9]. Herein, we report a case of GIST involving multiple intestinal areas associated with NF1 in black African adult.

\section{Case Presentation}

A 56-year-old man is referred from the gastroenterology to the surgery unit of the teaching hospital, Yopougon-Abidjan-Côte d'Ivoire, for recurrent intestinal bleeding and abdominal pain. His medical history revealed that his father had cutaneous neurofibromatosis. The patient did not take any anticoagulant medications nor suffer from any chronic liver disease. Physical examination showed a blood pressure of 90/50 and cardiac pulse of 110 beats per minute. Abdominal examination revealed a periumbilical pain without any abdominal palpable mass. Skin examination showed "café-au-lait" spots and cutaneous neurofibroma covering his trunk and arms (shown in Fig. 1). Blood test showed hemoglobin level of $4.2 \mathrm{~g} / \mathrm{dL}$ and C-reactive protein of $48 \mathrm{mg} / \mathrm{L}$. The patient received $2.8 \mathrm{~L}$ of blood transfusion in the next

Fig. 1. Cutaneous neurofibroma (+) and "caféau-lait" spots (++).

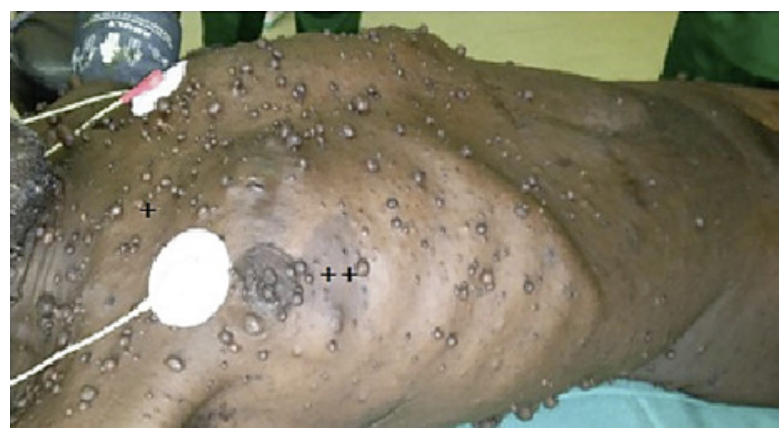


Fig. 2. CT scan showing intestinal wall thickness (a) and intra-abdominal nodules (b) with contrast enhancement.
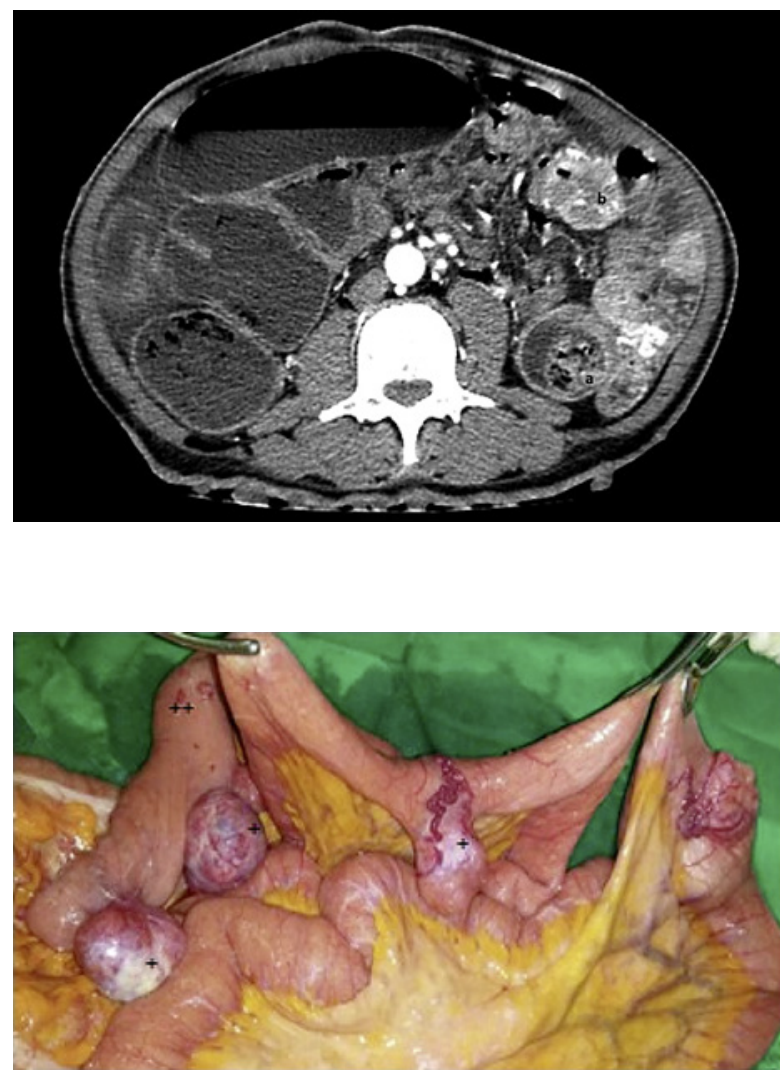

Fig. 3. Sizable nodules (+) on anti-mesenteric edge of small intestine and infra-centrimetric nodules $(++)$.

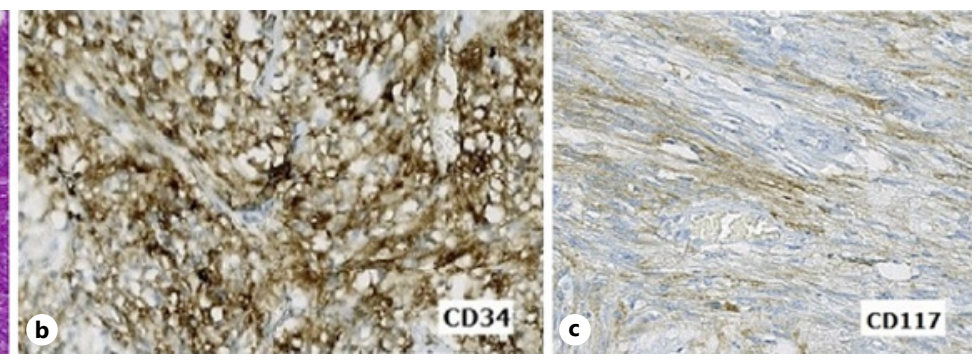

Fig. 4. a Dense fibrillar or epithelioid spindle cells of GIST associated with eosiniophilic cytoplasm and rare atypical cytonuclear without mitosis $(\mathrm{HES} \times 40)$. b Positive immunochemistry staining for CD34 $(\times 100)$. c Positive immunochemistry staining for CD117 $(\times 100)$. GIST, gastrointestinal stromal tumor.

5 days, which elevated his hemoglobin level to $12.8 \mathrm{~g} / \mathrm{dL}$. CT scan revealed several nodules located in the intestinal wall (Shown in Fig. 2a, b). Gastroscopy and colonoscopy were performed and revealed normal findings. However, exploratory laparatomy performed to investigate the recurrent intestinal bleeding showed multiple nodules in the antimesenteric intestinal wall edge, at 15, 40, 55, and $220 \mathrm{~cm}$ from the Treitz angle, with several micronodules (Shown in Fig. 3). Two resections and ileum-jejunum anastomosis were performed after removing several nodules. Histopathological examination of the nodules showed typical lesions of GIST and positive immunohistochemistry staining for CD34 and CD117 (Shown in Fig. $4 \mathrm{a}-\mathrm{c}$ ). The patient felt better with a normalized hemoglobin level of $12.8 \mathrm{~g} / \mathrm{dL}$. He was discharged 8 days after surgical treatment. He has not presented any rebleeding. 


\section{Discussion/Conclusion}

GIST is the most common mesenchymal tumor derived from interstitial cells of Cajal with a low mitotic index grade [1]. GIST occurring in a patient with NF1 differs from the sporadic one, which involves the stomach and duodenum [3]. In our case, the clinical feature of GIST was similar to that of previous case reports according to age of discovery and multiple small intestinal wall locations [3-5]. Intestinal involvement in GIST is one of the serious complications when associated with NF1 [6], that is, $70 \%$ of patients involving the small intestines and $<2 \%$ involving the colon [3]. GIST associated with NF1 rarely occurs; however, recurrent bleeding is the most common clinical symptoms for diagnosis in $27.5 \%$ of patients, in whom the origin is often missed by a gastrointestinal endoscopy and often leads to an emergency laparotomy [3, 5]. Previous reports demonstrated that emergency laparotomy should be performed in case of gastrointestinal bleeding with negative exploratory endoscopic examination, a more accurate procedure $[5,10]$. In our case, only CT scan was performed to evaluate the intestinal bleeding origin by showing the presence of nodularities on the intestine wall with contrast enhancement. Exploratory laparotomy revealed intestinal lesions, suggesting the presence of GIST associated with NF1 and confirmed by histolopathological examination with immunochemistry staining. Clinical manifestations of GIST in a patient with NF1 vary from abdominal pain, intestinal occlusion, intussusceptions, perforation, or bleeding resulting in chronic anemia $[3,4]$. Abdominal tumor may be found at physical examination [6]. NF1 is the most common characteristic of all neurofibromatosis diseases [2]. Our case was diagnosed based on the following criteria: the presence of skin spots and the family history of similar disease that allowed us to clinically establish the NF1 diagnosis [8]. GIST and NF1 do not share the same genetic mutation. Patients with sporadic GIST harbor the KIT or platelet derived-growth factor receptor, alpha tyrosine kinase protein mutation, which is rarely found in GIST associated with NF1 [2, 7, 11]. However, NF1 genetic mutation has been described in GIST patients without clinical manifestations [12]. Given the divergent genetic mutations between NF1 and GIST, an alternative pathway is hypothesized on the key role of GIST pathogenesis associated with NF1, implying the activation of the ras pro-oncogene or other similar pro-oncogene signal transduction pathways [7, 13]. Furthermore, various intestinal tumors involvements of NF1 are described, and immunochemistry analysis is mandatory to confirm the presence of GIST. GIST mostly express CD34 and CD117 when intestinal nodules are found to be associated with physical lesions of NF1 $[6,7]$. The most appropriate surgical treatment consisted of removing a large part of intestine involved in patient with intestinal bleeding [5]. Chemotherapy using imatinib, a tyrosine kinase inhibitor, is less effective in patients with GIST associated with NF1 with regard to the underlying genomic alteration that differs from sporadic GIST $[6,11]$. However, the risk of intestinal tumor recurrence remains in our patient because a few small tiny nodules have not been removed.

Practitioners in sub-Saharan Africa should consider GIST when the patient presents a recurrent intestinal bleeding associated with NF1 as clinical manifestations. Appropriate investigations should be made for better and efficient surgical treatment to prevent recurrences.

\section{Acknowledgements}

The authors would like to thank les laboratories CERBA, Pasteur, Cergy Pontoise (France) and Longchamp, Abidjan (Côte d'Ivoire), for providing the images of immunochemistry analysis.

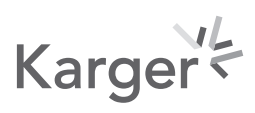




\section{Statement of Ethics}

The case report has been conducted in accordance with the Helsinky declaration. This case report was approved by the ethic committee of the Yopougon Teaching Hospital (Direction Médicale et Scientifique), approval number No. 006/MSHP/CHUY/DMS/okt/ $y f / 21$. Written informed consent was obtained from the patient for publication of the details of his medical case and any accompanying images.

\section{Conflict of Interest Statement}

The authors declare no conflict of interest regarding the manuscript.

\section{Funding Sources}

No financial support regarding the publication of the manuscript.

\section{Author Contributions}

Gnangoran Marcellin Koffi, provided the data, wrote the manuscript, and revised the final version. Alassan Mahassadi provided the data, wrote the manuscript, and the English transcription. Sylvanus Koui provided the first histological description of the lesions and wrote the manuscript. Kountele Gona Soro revised the final version of the manuscript. All authors mentioned above are accountable for all aspects of this case report.

\section{Data Availability Statement}

Data supporting this case report are freely and openly available in https://b2share.eudat. eu/records/c3c37cbffff34049baa690035572295e.

\section{References}

1 Søreide K, Sandvik OM, Søreide JA, Giljaca V, Jureckova A, Bulusu VR. Global epidemiology of gastrointestinal stromal tumours (GIST): a systematic review of population-based cohort studies. Cancer Epidemiol. 2016;40:39-46.

2 Rasmussen SA, Friedman JM. NF1 gene and neurofibromatosis 1. Am J Epidemiol. 2000;151(1):33-40.

3 Salvi PF, Lorenzon L, Caterino S, Antolino L, Antonelli MS, Balducci G. Gastrointestinal stromal tumors associated with neurofibromatosis 1: a single centre experience and systematic review of the literature Including 252 Cases. Int J Surg Oncol. 2013;2013:398570.

4 Wang M, Xu J, Zhang Y, Tu L, Qiu WQ, Wang CJ, et al. Gastrointestinal stromal tumors: 15-years'experience in a single center. BMC Surg. 2014;14:93.

5 Kim MS, Woo IT, Jo YM, Lee JH, Park BS. Life-threatening bleeding with intussusception due to gastrointestinal stromal tumor: a case report. Surg Case Rep. 2019;5:154.

6 Agaimy A, Vassos N, Croner RS. Gastrointestinal manifestations of neurofibromatosis type 1 (Recklinghausen's disease): clinicopathological spectrum with pathogenetic considerations. Inter J Clin Exp Pathol. 2012;5(9): 852-62.

7 Miettinen M, Jerzy Lasota J. Gastrointestinal stromal tumors. Review on morphology, molecular pathology, prognosis, and differential diagnosis. Arch Pathol Lab Med. 2006;130:1466-78.

8 Tonsgard JH. Clinical manifestations and management of neurofibromatosis type 1. Semin Pediatr Neurol. 2006;13(1):2-7.

9 Odebode TO, Afolayan EA, Adigun IA, Daramola 00. Clinicopathological study of neurofibromatosis type 1: an experience in Nigeria. Inter J Dermatol. 2005;44:116-20.

\section{Karger'}


10 Levy AD, Patel N, Dow N, Abbott RM, Miettinen M, Sobin LH. From the archives of the AFIP: abdominal neoplasms in patients with neurofibromatosis type 1: radiologic-pathologic correlation. Radiographics. 2005; 25(2):455-80.

11 Mussi C, Schildhaus HU, Gronchi A, Wardelmann E, Hohenberger P. Therapeutic consequences from molecular biology for gastrointestinal stromal tumor patients affected by neurofibromatosis type 1. Clin Cancer Res. 2008;14(14):4550-5.

12 Gasparotto D, Rossi S, Polano M, Tamborini E, Lorenzetto E, Sbaraglia M, et al. Quadruple-negative GIST is a sentinel for unrecognized neurofibromatosis type 1 syndrome. Clin Cancer Res. 2017;23(1):273-82.

13 Duensing A, Medeiros F, McConarty B, Joseph NE, Panigrahy D, Singer S, et al. Mechanisms of oncogenic KIT signal transduction in primary gastrointestinal stromal tumors (GISTs). Oncogene. 2004;23(22):3999-4006. 